\title{
Application of x-ray imaging to current profile measurements in the PEGASUS experiment
}

\author{
K. Tritz, R. Fonck, and T. Thorson \\ Department of Engineering Physics, University of Wisconsin-Madison, Madison, Wisconsin 53706
}

(Presented on 9 June 1998)

\begin{abstract}
For low-aspect ratio toroidal devices, flux shape information can be used as a constraint for reconstruction of the plasma current profile and $q$ profile. A model current profile was used to compare the sensitivity of the equilibrium reconstruction using soft X-ray (SXR) image constraints to motional Stark effect (MSE) constraints. The deviation in the $\chi^{2}$ of the fit versus current profile was similar in both cases, showing that the SXR data is as good a constraint on the equilibrium as MSE information. There are two soft x-ray imaging systems under consideration to determine the shape of the internal flux surfaces on PEGASUS. One diagnostic consists of a series of vertically spaced, tangentially viewing linear detector arrays. Another design using a two-dimensional pinhole camera for a soft x-ray image of the plasma is also being evaluated. (C) 1999 American Institute of Physics. [S0034-6748(99)57801-5]
\end{abstract}

\section{INTRODUCTION}

The determination of the local current density in magnetically confined plasmas is a critical diagnostic for most toroidal configurations, and is especially important for the next generation of high-beta experiments such as low-aspect ratio tokamaks, spheromaks, and related configurations. For large, high-field tokamaks several diagnostic techniques for current measurements have evolved, and the motional Stark effect (MSE) diagnostic has emerged as the standard for localized measurements of the poloidal field, allowing detailed understanding of the current density profile and its role in achieving advanced performance regimes. However, a new generation of plasma experiments will concentrate studies of plasmas in alternate magnetic geometries and these usually employ low magnetic fields and compact geometry without the benefit of large high-power heating neutral beams. Hence, there is a renewed need to develop relatively simple, inexpensive means of determining the plasma magnetic equilibrium.

This article discusses the use of $\mathrm{x}$-ray imaging of plasma flux surfaces in low-aspect ratio tokamaks for determination of the plasma current density profile. In particular, it considers application of this technique to the new PEGASUS extremely low aspect ratio toroidal experiment, but similar considerations apply to the new large spherical tokamaks such as National Spherical Torus Experiment (NSTX) and Mega Ampere Spherical Tokamak (MAST). A soft x-ray imaging diagnostic provides an alternative which can take advantage of the strong shaping inherent in spherical tokamaks. The soft x-ray emissivity can be used as constraints in an equilibrium code, just as MSE data is used, to reconstruct the current profile of the plasma equilibrium.

The concept of using flux surface shape information as a method of current profile determination was first proposed by Christiansen and Taylor. ${ }^{1}$ It was shown that if the surfaces were represented by some function $F(R, Z)=$ constant, then the current distribution could be represented by a function
$\lambda(F)$, which was determined on each surface from the gradient and Laplacian of $F$. Because $\lambda(F)$ was related to the change in $\nabla F$ around the surface, this technique broke down in the limit of large aspect-ratio and circular flux surfaces, but can be quite useful in diagnosing highly noncircular plasmas, such as those found in low-aspect ratio tori. The high amount of redundant information in $F$ created an overdetermined system which can be used to numerically reconstruct a most likely "least-squares" current profile even with noisy data.

Assuming that soft $\mathrm{x}$-ray emission is constant on a flux surface, surfaces of constant emissivity can be interpreted as flux surfaces. Attempts to directly measure the flux surface shape, and hence infer the plasma current distribution, via tomographic inversions of $\mathrm{x}$-ray intensities from crossed one-dimensional imaging cameras have met with mixed results. Typically, determination of the shapes of the flux surfaces have proved difficult due to the sensitivities of the inversion process.

Tomographic inversion of soft x-ray intensity measurements was used on the Joint European Torus (JET) tokamak to determine the geometric shift and ellipticity of the internal flux surfaces. ${ }^{2}$ These moments were used to determine the various parameters of the plasma equilibrium, which were then compared with the results from an equilibrium reconstruction using only external magnetic diagnostic information. The results of the comparison between the two methods showed good agreement through $0.2<r / a<0.8$ of the plasma, but the reconstruction was not sensitive enough to accurately determine $q(0)$ to within $10 \%$. In contrast, direct inversion of $\mathrm{x}$-ray emission contours on Alcator C-mod has inferred strong deviations of the contours of constant emissivity from flux surface shape inferred from external magnetics measurements of the plasma equilibrium. ${ }^{3}$

To avoid the difficulties with direct image inversions, profile reconstruction using soft $\mathrm{x}$-ray images was pursued on the Princeton Beta Experiment (PBX) using a forward 
modeling technique, with reasonable success. ${ }^{4,5}$ This technique avoided the inherent numerical uncertainty in a tomographic inversion, and consisted of fitting a soft $\mathrm{x}$-ray image, constructed by projecting the results of a magnetic equilibrium code, to the measured two-dimensional soft x-ray image of the plasma. The input parameter $q(0)$ was changed manually in the equilibrium reconstruction code until the $\chi^{2}$ of the fitted images was minimized. By avoiding tomographic inversion, the current profile was determined throughout the plasma and $q(0)$ was resolved to within $10 \%$. These results indicate that a potentially much more robust means of utilizing soft x-ray intensities to determine the plasma equilibrium consists of combining imaging data with all other relevant measurements (such as external flux, pickup coils, plasma current, etc.) of the plasma to internally constrain the equilibrium allowed by fitting to the basic toroidal equilibrium equation.

We note that no measurement technique provides a direct measure of the plasma current density in noncircular, paramagnetic plasmas such as those found in high-beta spherical tokamaks. Instead, all internal magnetic field diagnostics, including MSE and Faraday rotation, are employed as constraints to magnetic equilibrium fits. These constraints on the internal behavior of the plasma, along with boundary constraints from external magnetic diagnostics, are used by current equilibrium codes to determine the current profile of the plasma. Equilibrium codes generate solutions to the Grad-Shafranov equation which is derived from the steady state ideal magneto hydrodynamic (MHD) equations.

$$
\Delta * \psi=-\mu_{0} R J_{\phi}=-\mu_{0} R^{2} \frac{d p}{d \psi}-g \frac{d g}{d \psi} .
$$

The Grad-Shafranov equation is a second order partial differential equation that describes the tokamak equilibrium, where $p(\psi)$ represents the pressure and $g(\psi)$ represents the toroidal field. For typical profiles of $p$ and $g$, this equation must be solved numerically. The iterative calculations are constrained by various boundary conditions, and also by measurements for which the equilibrium code needs to find a self-consistent solution.

In this article, we discuss two x-ray imaging systems under consideration for deployment on the PEGASUS toroidal experiment for use in determining the plasma current profile. Two relatively simple imaging systems are considered for incorporation of the x-ray intensity information into the equilibrium reconstruction code itself. The equilibrium code will use the image comparison as an internal constraint to the equilibrium along with the other constraints provided by external magnetic diagnostics. The two soft $\mathrm{x}$-ray diagnostic variants presented here require slightly different analysis techniques, although both provide constraints to an equilibrium reconstruction code.

\section{IMPLEMENTATION}

Tokamac, an equilibrium code developed at Columbia University to solve the Grad-Shafranov equation, was used to evaluate the use of $\mathrm{x}$-ray images for equilibrium con- (a)

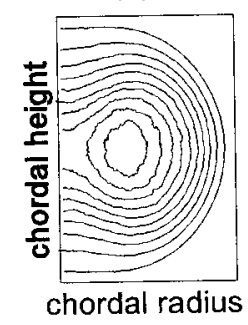

(d)

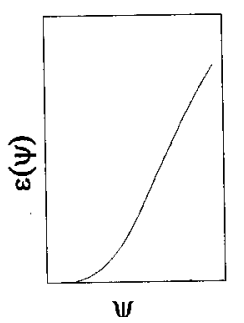

(b)

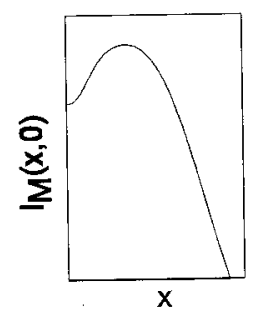

(e)

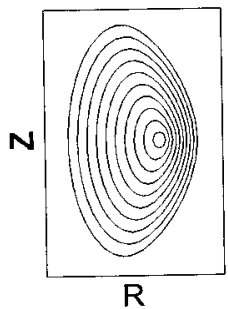

(c)

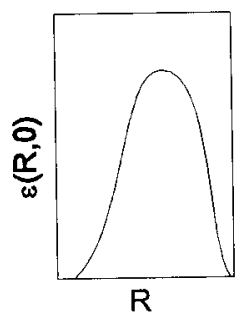

(f)

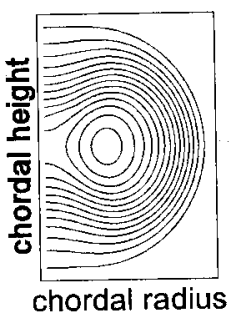

FIG. 1. Technique to constrain equilibrium code using SXR pinhole camera. (a) SXR intensity image from camera, (b) midplane of measured intensity image, (c) Abel-inversion of intensity to obtain emissivity profile, (d) emissivity mapped to a flux profile generated from equilibrium code, (e) map used to create model emissivity cross section, (f) model intensity projection generated to compare with (a).

straints using simulated measurements of SXR intensity profiles and simulated external magnetic measurements.

The simplest soft $\mathrm{x}$-ray diagnostic under development is similar to that used on PBX-M, and consists of a tangentially viewing charge-coupled device (CCD) pinhole camera which takes a two-dimensional image of the SXR intensity of the plasma. Unfortunately, the use of this information to constrain an equilibrium code is not straightforward.

First, the SXR intensity on the horizontal midplane of the pinhole is Abel-inverted to obtain an soft x-ray emissivity profile across the midplane of the plasma cross section. The midplane emissivity profile is then mapped to a flux surface profile generated from an iteration of an equilibrium code. This mapping is then used to generate a soft $\mathrm{x}$-ray emissivity image over the entire cross section of the plasma. The generated image is then projected toroidally to create a SXR intensity image to compare to the measure CCD image. A $\chi^{2}$ can then be calculated to determine how closely the two images match (see Fig. 1). While this process is numerically cumbersome, it should provide a strong constraint to the equilibrium reconstruction, and the simple tangentially viewing pinhole camera is also relatively easy, technically, to implement.

Limitations inherent in the current version of the equilibrium code used for the sensitivity modeling prevents ready simulation and fitting to this full two-dimensional pinhole camera image. Like almost all equilibrium codes, Tokamac employs a least squares linear fitting routine to fit to the measured quantities, which are assumed to be linear functions of the poloidal flux $\psi$. Because most magnetic measurements and the profiles are indeed linear functions of $\psi$, this method of solution has worked quite well. However, linear analysis using the two-dimensional image as a direct con- 


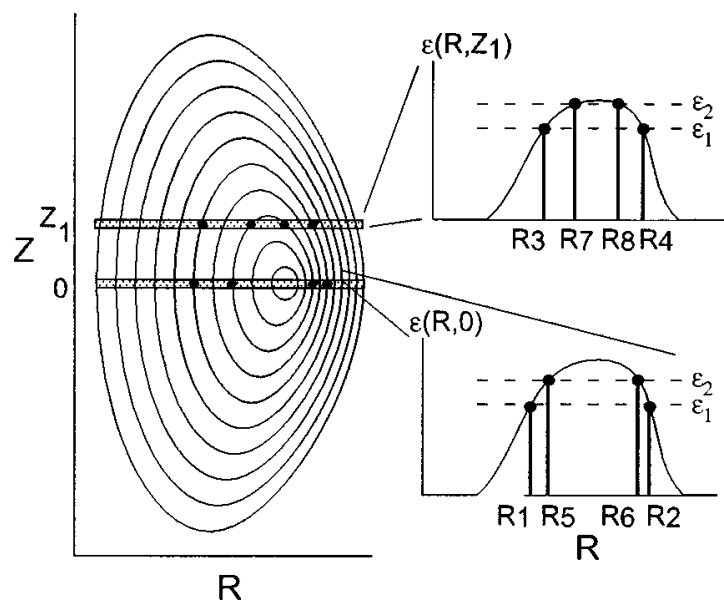

FIG. 2. Linear arrays of horizontal, tangentially viewing SXR detectors identify sets of coordinate pairs with equal emissivity, which correspond to points of equal flux.

straint requires restricting the image emissivity to a fixed functional form. To circumvent this problem on PBX, Powell manually inserted the assumed plasma profiles to obtain a minimum $\chi^{2}$ fit to the measured image. Such an approach is cumbersome and allows determination of a fit to only a minimum number of parameters. A nonlinear fitting method would allow much more flexibility in the both parameterization of the profiles, and the types of measurement constraints allowed in the reconstruction. We thus defer a detailed evaluation of the simple pinhole camera integration into the equilibrium code until further development of the basic equilibrium code is undertaken.

Nevertheless, the use of a linear fitting routine has the advantage of being relatively simple to implement and computationally quite efficient, and is adaptable to an extended arrays of one-dimensional tangentially viewing soft x-ray arrays. This second and somewhat more tractable SXR diagnostic approach consists of a series of tangentially viewing horizontal linear detector arrays spaced vertically apart, as indicated in Fig. 2. The soft x-ray intensity profiles from these detectors can be directly Abel-inverted, due to their cylindrical symmetry, resulting in emissivity profiles across the plasma cross-section at various vertical locations. Invoking the assumption that SXR emissivity is constant on a flux surface, coordinate pairs of equal emissivity can be identified to lie on the same flux surface. This flux surface information, along with external magnetic diagnostic measurements, is then used to constrain an equilibrium reconstruction. In particular, the metric given by the squared deviation of the flux at each coordinate pair from the average flux over the set of paired points,

$$
\delta_{i}=\frac{\sum_{j=1}^{N}\left[\psi_{i}\left(R_{i, j}, Z_{i, j}\right)-\bar{\psi}_{i}\right]^{2}}{\bar{\psi}_{i}^{2}}
$$

is minimized in a least squares sense in conjunction with all external magnetics measurements. The measured quantity, pairs of $\psi(R, Z)$, is directly applicable for use in the linear fitting method.

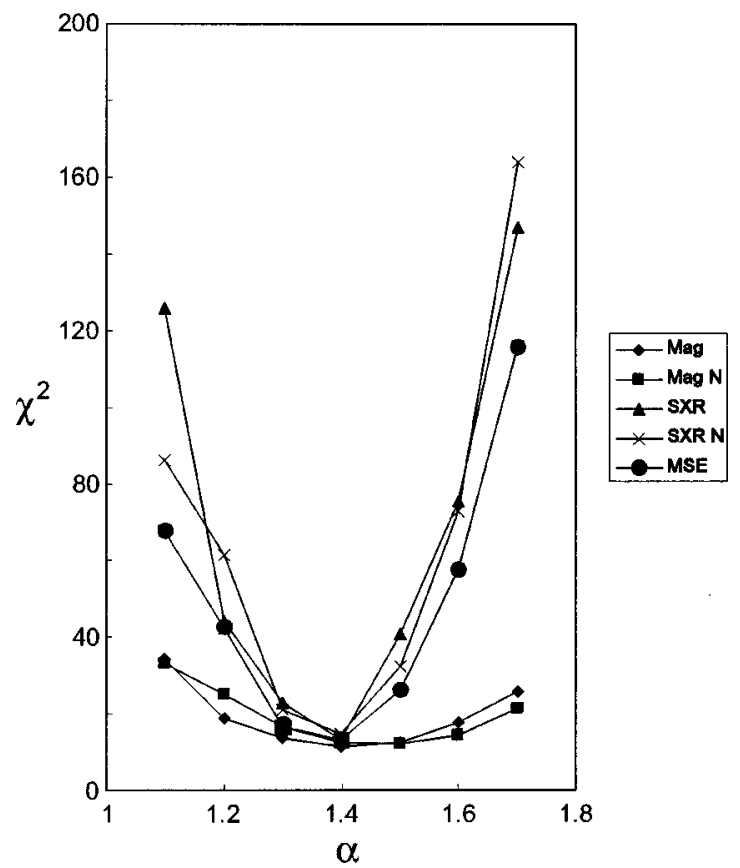

FIG. 3. Scan of $\chi^{2}$ as a function of profile peakedness parameter $\alpha$ for six constraint cases: external magnetics only, external magnetics with 5\% noise added, internal SXR constraints with external magnetics with and without $5 \%$ noise, and MSE constraints with external magnetics.

\section{SENSITIVITY TESTS}

To evaluate the potential for these SXR measurements to extract details of the current profile, model profiles were used to generate simulated SXR image data, which in turn were used to constrain the equilibrium fit to simulated magnetics data. Current and pressure profiles were specified to generate a baseline equilibrium. This equilibrium was then used to generate the simulated soft $\mathrm{x}$-ray intensity profiles and the external magnetic diagnostic information. In addition, localized MSE data was also used to constrain these fits, and hence allow a reasonably direct comparison of the sensitivity of the equilibrium fit to SXR image data and MSE poloidal field measurements.

The model current profile was varied to map the rms deviation of the measurements to the fitted values in $\chi^{2}$ to changes in profile shape. This was accomplished by varying the poloidal current term:

$$
g \frac{d g}{d \psi} \propto(1-\psi)^{1-\alpha}
$$

through variations in the peaking factor $\alpha$.

Figure 3 shows the variation in $\chi^{2}$ as a function of $\alpha$ for a case wherein the simulated data was obtained for a value of $\alpha=1$.4. Six separate curves are shown: one obtained using external measurements only, another using external measurements only with $5 \%$ random noise added to the simulated measurements, using both the external magnetics and the internal constraint derived from SXR equal-emissivity point pairs, using the same with $5 \%$ noise added, and finally using the simulated motional Stark effect measure of the local poloidal field and the external magnetics measurements. The 
internal MSE measurements were generated from the baseline equilibrium and used along with the external magnetics information to constrain the reconstruction.

The results show that the external magnetic constraints were relatively insensitive to the shape of the current profile, in that there is only a weak variation in the derived $\chi^{2}$ as a function of the profile peaking factor. With the addition of the SXR constraints, the $\chi^{2}$ of the reconstruction clearly shows a minimum at the expected profile shape. Next, the sensitivity of the SXR constraint was compared with a standard MSE constraint. The $\chi^{2}$ sensitivity of the reconstruction to the variation of the current profile is seen to be comparable between the simulated SXR intensity profiles and the simulated MSE measurements. Thus, the SXR-derived constraints on the deduced current profile are equivalent in sensitivity to that imposed by the standard MSE diagnostic measurement.

\section{DISCUSSION}

Finally, modeling was done to determine if a plasma with typical PEGASUS parameters would result in a large enough soft $\mathrm{x}$-ray intensity signal to minimize photon noise and other uncertainties in the pinhole systems. A temperature and density profile was derived from a pressure profile generated by the equilibrium code. The peak electron temperature was assumed to be. $5 \mathrm{keV}$, and the peak electron density was taken as $5 \times 10^{19} \mathrm{~m}^{-3}$. These profiles, along with an assumed impurity concentration of 3\% carbon, 3\% oxygen, and $0.1 \%$ iron, were input into a code which output an x-ray energy emission spectrum for each point in the profile. The x-ray mapping was then used to generate a plasma cross section emissivity which was projected onto a model CCD intensity image. The modeling shows that for a $10 \mathrm{~ms}$ integration time, the photon noise expected is $<1 \%$.

A necessary next step in these evaluations is the incorporation of a nonlinear fitting routine into the equilibrium code to verify that the two-dimensional pinhole camera is a strong enough constraint to help reconstruct details of the current profile. Performing profile sensitivity modeling of the two-dimensional pinhole camera with the modified code will specify the signal levels required from the hardware to perform accurate equilibrium reconstructions. These signal levels will determine the final geometry of the pinhole camera to be installed on the PEGASUS toroidal experiment.

Flux shape information has been shown to be a strong constraint on current profile reconstruction. Soft x-ray imaging shows promise as a relatively easy profile diagnostic to implement, and is well suited to spherical torus experiments. Profile reconstruction using SXR data as a constraint has been shown to work on past experiments, such as PBX, and is planned as a profile diagnostic on PEGASUS and NSTX. Modeling has been done to show that simulated soft X-ray data from linear arrays, along with external magnetic diagnostic information, does indeed provide enough information about the interior plasma region to allow accurate profile reconstruction with an equilibrium code. Using soft x-ray imaging to gain information about the shapes of the flux surfaces is relatively inexpensive and simple way to exploit the features of spherical tori, such as highly noncircular flux surfaces, while avoiding some of the inherent pitfalls of trying to use standard current profile diagnostics on low aspectratio experiments.

\section{ACKNOWLEDGMENT}

This work was supported by the U.S. DOE Grant No. DE-FG0296ER54375.

${ }^{1}$ J. P. Christiansen and J. B. Taylor, Nucl. Fusion 22, 111 (1982).

${ }^{2}$ J. P. Christiansen, J. D. Callen, J. J. Ellis, and R. S. Granetz, Nucl. Fusion 29, 703 (1989).

${ }^{3}$ M. C. Borrás and R. S. Granetz, Plasma Phys. Controlled Fusion 38, 289 (1996).

${ }^{4}$ E. T. Powell, R. J. Fonck, R. Kaita, N. Asakura, R. E. Bell, S. M. Kaye, H. W. Kugel, B. LeBlanc, F. M. Levinton, M. Okabayashi, S. F. Paul, D. W. Roberts, S. Sesnic, and H. Takahashi, Nucl. Fusion 33, 1493 (1993).

${ }^{5}$ A. Holland, R. J. Fonck, E. T. Powell, and S. Sesnic, Rev. Sci. Instrum. 59, 1819 (1988). 